\title{
Potential and limitations of wave intensity analysis in coronary arteries
}

\author{
Maria Siebes · Christina Kolyva $\cdot$ Bart-Jan Verhoeff • \\ Jan J. Piek $\cdot$ Jos A. Spaan
}

Received: 4 January 2009/Accepted: 16 January 2009/Published online: 10 February 2009

(C) The Author(s) 2009. This article is published with open access at Springerlink.com

\begin{abstract}
Wave intensity analysis (WIA) is beginning to be applied to the coronary circulation both to better understand coronary physiology and as a diagnostic tool. Separation of wave intensity (WI) into forward and backward traveling components requires knowledge of pulse wave velocity at the point of measurement, which at present cannot accurately be determined in human coronary vessels. This prompted us to study the sensitivity of wave separation to variations in wave speed. An estimate of wave speed ( $\mathrm{SPc})$ was calculated based on measured distal intracoronary pressure and Doppler velocity in normal and diseased coronary vessels of patients during hyperemia. Changes of the area under separated WI waveforms were determined for a range of wave speeds from 25 to $200 \%$ of the calculated value. Variations in wave speed between half to twice the calculated value did not substantially alter separated WI. In conclusion, although SPc lacks accuracy in determining local coronary wave speed it is within limits still applicable for wave separation in coronary WIA.
\end{abstract}

M. Siebes $(\bowtie) \cdot$ C. Kolyva · J. A. Spaan

Department of Biomedical Engineering and Physics, Academic Medical Center, University of Amsterdam, Meibergdreef 9, 1105 AZ Amsterdam, The Netherlands e-mail:m.siebes@amc.uva.nl

B.-J. Verhoeff · J. J. Piek

Department of Cardiology, Academic Medical Center, University of Amsterdam, Amsterdam, The Netherlands

Present Address:

C. Kolyva

Brunel Institute for Bioengineering, Brunel University, Uxbridge, UK
Keywords Wave intensity analysis .

Coronary circulation $\cdot$ Stenosis $\cdot$ Arterial wave speed

\section{Introduction}

Wave intensity analysis (WIA) has become a useful method for evaluating traveling pressure and flow waves in the aorta and larger arteries in the time domain. An in-depth overview of WIA and its application is provided elsewhere [15, 16] including this special issue [17, 23]. Wave patterns analyzed by WIA provide information about events originating upstream and downstream of the measurement site and WIA has great potential to improve our understanding of coronary-cardiac interaction [2, 4, 24, 25].

In the epicardial coronary arteries a forward compression and forward expansion wave can be recognized as in the aorta. However, specific to the coronary system are a backward compression wave early in systole and backward expansion wave at the time of aortic valve closure. These backward waves result from the onset of myocardial contraction and relaxation and represent a novel parameter to study the interaction of heart contraction and coronary flow. It is important to recognize that waves in the coronary circulation are actively generated by both upstream and downstream events [24]. However, separation of net waves into their forward and backward components requires knowledge of wave speed, which is difficult to measure in the coronary arteries $[9,16]$.

Note that we use the following terminology to identify the nature of the waves $[2,16]$ : Compression waves cause an increase in pressure, while expansion waves serve to decrease pressure. Pressure and velocity change together in forward waves, but in opposite ways in backward waves. 
Although a method has been proposed to estimate coronary wave speed in humans [3], there are complications in its application to these relative short arteries. Arterial pulse wave velocity is commonly in the order of $10 \mathrm{~m} / \mathrm{s}$ and the dominant frequency content of the pressure and flow velocity signals in the coronary system is below $50 \mathrm{~Hz}$. Hence, the smallest wavelength of the corresponding relevant wave component is several times longer than the length of a human coronary artery. One may therefore expect that pressure in the epicardial coronary vessels is rapidly equalized to such an extent that one can hardly speak of traveling waves. Conceptually, a wave also travels in a short vessel and one may consider the short vessel as a part of a longer tube, but along a short vessel there will hardly be a longitudinal gradient in pressure. We have therefore previously argued that a windkessel model would be more appropriate for the coronary artery system than a transmission line model [12].

At present there is no practical solution to the problem of measuring wave speed in coronary arteries, especially in humans. In the aorta several methods exist to determine wave speed, the foot-to-foot method and two single-point methods, the slope of the pressure-velocity curve in early systole (PU-loop) [9-11] and the sum-of-squares technique [3]. However, the first two are not feasible in the coronary arteries and the sum-of-squares technique is dominated by low-frequency characteristics of coronary waveforms and fails to produce reliable results in the coronary artery [12].

The purpose of this paper is to discuss the potential and the limitations of WIA and wave separation in the coronary arteries and focuses on the problems arising from lack of knowledge of coronary wave speed. We will do so on some examples of clinical data.

\section{Methods}

\subsection{Hemodynamic measurements}

Data for this study were derived from existing pressure and velocity recordings acquired in patients during cardiac catheterization. The dataset included hemodynamic measurements obtained in an angiographically normal reference vessel and in a diseased coronary artery before and after revascularization by stent placement. All measurements in the diseased artery were taken in an apparently normal vessel segment sufficiently downstream of the stenosis or stent to avoid flow disturbances. Epicardial vessel tone was relaxed by intracoronary administration of $0.1 \mathrm{mg}$ nitroglycerin.

Aortic pressure was measured via the guiding catheter at the coronary ostium and a 0.014-inch dual-sensor guide wire (Volcano Corp., Rancho Cordova, CA, USA) was advanced to a distal position in the interrogated coronary vessel to simultaneously record coronary perfusion pressure $(P)$ and flow velocity $(U)$ signals $[19,27]$. Care was taken to measure at the same downstream location before and after stent placement. All data presented here were collected during the hyperemic response to an intracoronary bolus of adenosine and were digitized together with the ECG at $120 \mathrm{~Hz}$ for off-line analysis.

The protocol was approved by the medical ethics committee of our institution and all patients gave written informed consent.

\subsection{Data analysis}

Data processing was performed using custom software (Delphi version 6.0, Borland Software Corporation, Cupertino, CA, USA) as described previously [12]. Smoothed derivates of the coronary $P$ and $U$ signals obtained by applying the Savitzky-Golay filter [18] were used to derive a single-point estimate of the local coronary wave speed, SPc, according to the sum-of-squares method as described in Ref. [3]:

$\mathrm{SPc}=\frac{1}{\rho} \sqrt{\frac{\sum \mathrm{d} P^{2}}{\sum \mathrm{d} U^{2}}}$

A value of $\rho=1,050 \mathrm{~kg} / \mathrm{m}^{3}$ was used for blood density and summations were taken over an integer number of cardiac periods.

Separated forward and backward wave intensity (WI) components were calculated for ensemble-averaged beats according to Ref. [24]:

$\mathrm{WI}_{ \pm}= \pm \frac{1}{4 \rho c}\left(\frac{\mathrm{d} P}{\mathrm{~d} t} \pm \rho c \frac{\mathrm{d} U}{\mathrm{~d} t}\right)^{2}$

resulting in WI expressed as $\mathrm{W} /\left(\mathrm{m}^{2} \mathrm{~s}^{2}\right)$, normalized for the sampling rate by utilizing the time derivatives of $P$ and $U$. Wave energy was calculated as the area under the curve for forward (positive) and backward (negative) WI, respectively. The ratio of forward and backward wave energies of the ensembled cycle was used to assess the relative contribution of upstream and downstream generated waves.

\subsection{Simulations of wave speed variability}

The extent to which the summed forward and backward wave energies depended on wave speed was examined for hyperemic data obtained in three patients with different sum-of-squares estimates of wave speed. Wave speed was varied from 25 to $200 \%$ of the corresponding value assessed from the sum-of-squares technique. 
Table 1 Coronary vessel, diameter stenosis and hyperemic wave speed estimate of three patients selected as examples

\begin{tabular}{lllllc}
\hline Diseased artery & Diameter stenosis $(\%)$ & SPc 'stenosis' $(\mathrm{m} / \mathrm{s})$ & SPc 'stent' $(\mathrm{m} / \mathrm{s})$ & Reference artery & SPc 'ref' $(\mathrm{m} / \mathrm{s})$ \\
\hline RCA & 66 & 57 & 14 & LCX & 10 \\
RCA & 62 & 33 & 9 & LAD & 13 \\
LAD & 21 & 7 & LCX & 11 \\
\hline
\end{tabular}

$L C X$ left circumflex artery, $L A D$ left anterior descending artery, $R C A$ right coronary artery, $S P c$ single-point wave speed obtained by the sum-ofsquares method (Eq. 1)
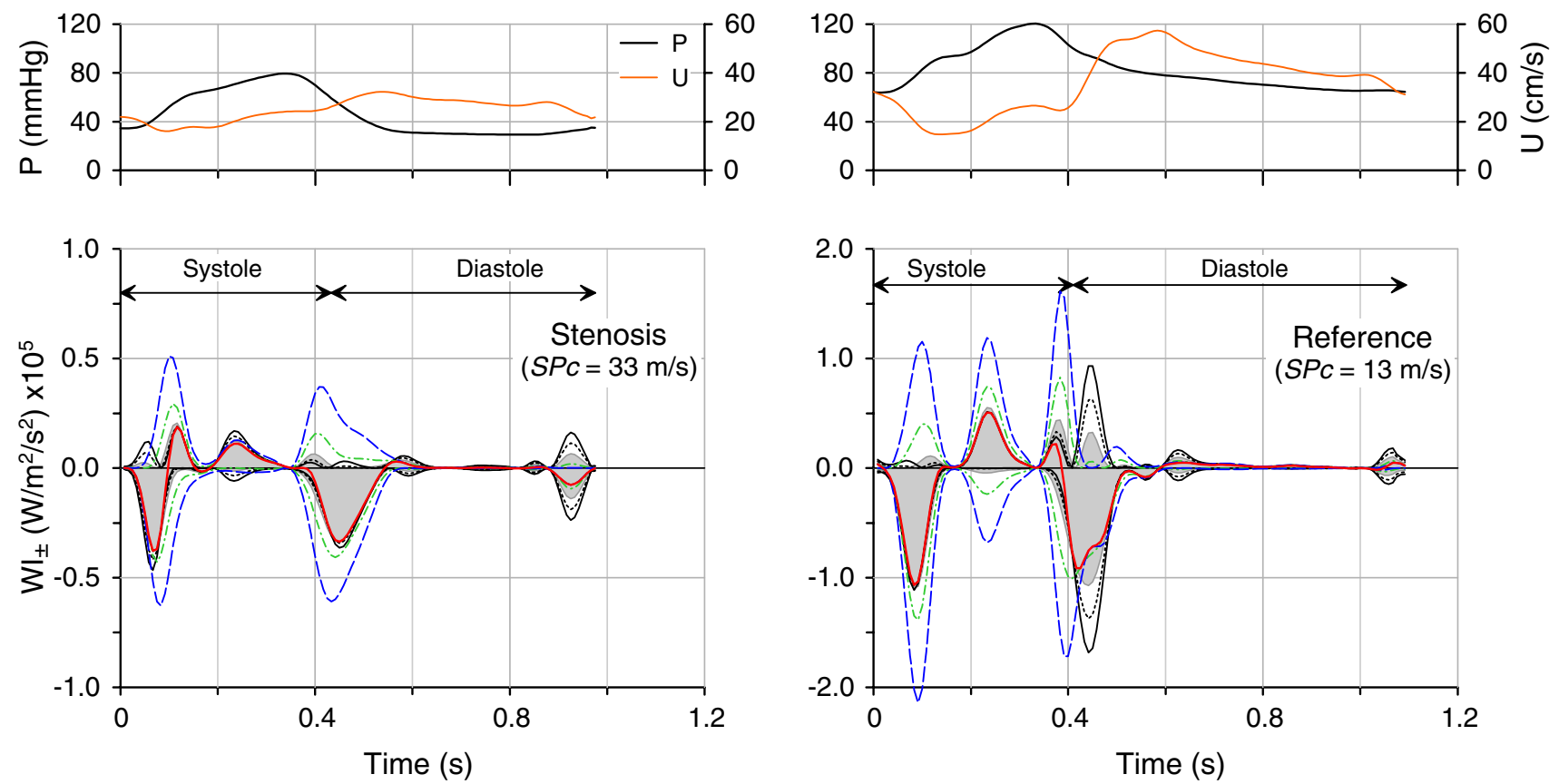

Fig. 1 Wave intensity (WI) from the diseased right coronary artery (62\% diameter stenosis proximal to measurement site; left) and the healthy left anterior descending coronary artery (right) of a 62-yearold patient, separated into forward (WI $>0$ ) and backward $(\mathrm{WI}<0)$ components. The actual pressure and flow velocity signals are provided in the sub-panels. Separated WI is displayed for the wave

\section{Results}

Wave speeds calculated on the full data set were reported earlier [12]. For the present report and sake of the discussion, we selected three patients covering a wide range of hyperemic wave speed estimates by the sum-of-square method in the presence of a discrete proximal stenosis: 21,33 and $57 \mathrm{~m} / \mathrm{s}$. The wave speed estimates in the reference vessel of these patients and in the target vessel after stent placement were considerably lower and averaged $10.7 \pm 2.6 \mathrm{~m} / \mathrm{s}$. Individual values per patient and vessel condition are provided in Table 1 and in the bottom panels of Fig. 2 .

Figure 1 illustrates the effect of variations in wave speed on separated WI patterns for a patient with hyperemic $\mathrm{SPc}=33 \mathrm{~m} / \mathrm{s}$ in a coronary artery segment downstream of

speed estimate calculated with the sum-of-squares technique (calculated SPc, shaded areas) and for simulated variations ranging from 25 to $200 \%$ of this value. Data are from an ensemble-averaged beat during microvascular vasodilation. The red waveform represents the net wave intensity. Note that the scale for WI of the reference vessel is twice that of the stenotic vessel

a $62 \%$ diameter stenosis (left) and in the reference vessel (right) of the same patient. Wave intensities are shown for an ensemble-averaged heart cycle, starting with the R-peak of the ECG. The effect of varying wave speed from one quarter to twice its estimated value (shaded in gray) on separated forward and backward WI is shown. The waves at the end of diastole are self-canceling and may be considered the result of an artifact. These artifacts were reduced by a simulated decrease in wave speed and enlarged when wave speed was increased. No additional self-canceling waves were induced nor was the WI profile or timing during the heart cycle substantially altered when SPc deviated in a positive direction, except in early diastole. Lowering SPc had a larger influence on the separated waves, increasing both forward and backward wave 
Fig. 2 Total wave energy carried during the heart cycle by forward $(F$, top panels $)$ and backward traveling $(B$, middle panels) waves, and their ratio (B/F, bottom panels). Results during hyperemia are shown for different percent deviations from the wave speed calculated with the sum-of-squares singlepoint technique. The selected examples from three patients cover different wave speeds (indicated by numbers in $B / F$ panels), percent diameter stenoses (DS) and vessels. Separated wave energies markedly increase when wave speed is lowered to less than half of the calculated value. The $\mathrm{B} / \mathrm{F}$ ratio is closer to unity in the absence of a stenosis and for physiological values of wave speed
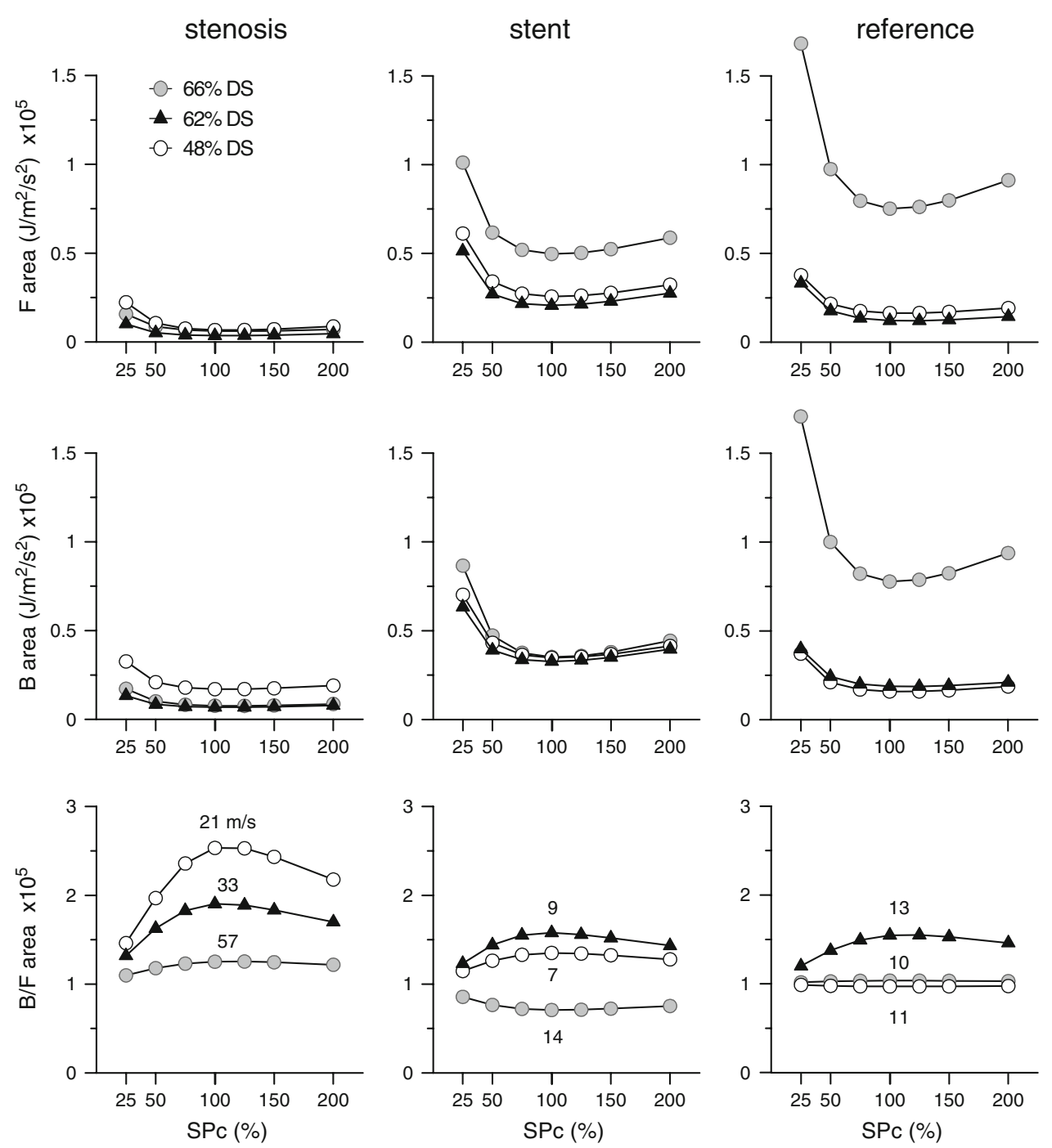

components at the beginning of the cycle. Note that the energy in the waves downstream of a stenosis is about a half of that in the reference vessel.

In order to quantify these changes in WI, we determined the integrated backward (B) and forward (F) wave area, representing the total energy during a complete heart cycle carried by backward and forward waves, respectively, and their ratio (B/F area). Results are shown in Fig. 2 for the three selected patients. Wave speed was lower and the $\mathrm{B} / \mathrm{F}$ ratio (bottom panels) was closer to unity after stent placement and in the reference vessels than in the stenotic vessels. For wave speed variations within $\pm 25 \%$, the increase in forward wave energy was on average not higher than $5.5 \%$ and backward wave energy increased $<3.5 \%$. This led to a change in the $\mathrm{B} / \mathrm{F}$ ratio by $2.3 \%$ in stenotic vessels, $0.4 \%$ after revascularization and $0.5 \%$ in the reference vessels. For larger variations in wave speed, the energy of the separated forward and backward waves was more sensitive to reductions of the initial single-point estimate, especially for the forward waves.

Lowering wave speed also affected the $\mathrm{B} / \mathrm{F}$ ratios to a larger extent than raising it and this effect was more pronounced the more it differed from unity at the initial wave speed estimate (lower left panel, stenosis). In all cases, the $\mathrm{B} / \mathrm{F}$ ratio approached a value of 1 by lowering wave speed.

\section{Discussion}

4.1 Potential of WIA for the interpretation of coronary pressure and flow velocity signals

The specific nature of pulsatile coronary flow and pressure waveforms has been known for a long time [7]. The intramyocardial pump model provided a biomechanical basis for the observation that coronary systolic flow is 
lower than diastolic flow despite the higher aortic pressure in this period [21]. The lower systolic flow was explained by the squeeze of the intramural vessels induced by systolic compression, thereby imposing a retrograde flow component to the net forward flow when averaged over the whole heart beat. However, it became clear that this interpretation had to be modified to include time-dependent variations of contractile parameters like myocardial elastance $[13,28]$. The varying elastance concept was applied to explain coronary systolic flow impediment [14, 22]. The problem was, however, how to interpret coronary pressure and flow signals in concert while both are varying in time and mutually affecting each other. It is at this point that WIA has come to the rescue since it established a new definition of waves combining the pressure and flow velocity signal into a new biomechanical parameter.

The potential of WIA for the coronary circulation lies in its ability to distinguish the effects of proximal and distal mechanical events on the coronary pressure and flow signals. These relationships may provide unique possibilities in characterizing and perhaps in the end diagnosing several types of coronary epicardial and microvascular disease [4].

The physiological events that determine the WIA patterns in the coronary arteries can be distinguished schematically by four phases [24]. (A) The first phase is the period of isovolumic contraction of the heart. The aortic pressure still exhibits its diastolic form and only varies slowly at this time. However, the increase in wall tension squeezes the intramural vessels and generates a backward compression wave. (B) The second phase is also in systole but just after aortic valve opening. In this phase elastance of the wall has increased thereby protecting the intramural vessels from further compression by the resulting increase of left ventricular pressure [13]. However, aortic pressure is rising sharply and sends a forward compression wave into the epicardial coronary arteries. (C) In the third phase, the decay of left ventricular elastance allows a forward wave into the coronaries through muscle relaxation. This results in a forward expansion wave. (D) The decrease in aortic pressure after valve closure will try to reduce coronary vascular filling and drives a backward expansion wave. The problem with the interpretation of WIA is that these four physiological events and corresponding net waves are not fully separated in time. Especially events A and B may overlap as well as C and D. In theory (Eq. 2), net waves can be separated as shown in Fig. 1, but for doing so the coronary wave speed is needed.

\subsection{Applicability of coronary wave separation in humans}

The single-point method based on the sum-of-squares (Eq. 1) is the only practically applicable technique to estimate coronary wave speed in humans [3]. However, despite its sound theoretical foundation in WIA theory, this method still faces some conceptual problems. When applied to data obtained in patients we discovered dependencies that are hard to understand on the basis of physiological knowledge [12]. After stenosis revascularization by angioplasty and stent placement, distal pressure was restored to normal levels, but wave speed assessed in the downstream vessel segment markedly decreased (see also SPc values in Table 1). This puzzling observation is opposite to what would be expected based on the positive relation between wave speed and mean coronary distending pressure demonstrated in dogs [1]. Moreover, when a microvascular dilator was administered that does not affect smooth muscle tone in the larger coronary arteries, wave speed decreased by a factor of two while it should have remained constant. Hence, at present we lack a trustworthy method for the determination of coronary wave speed while it is needed for wave separation.

It is outside the scope of this paper to analyze the mathematical and physical issues that result in the nonphysiological predictions of wave speed. However, the lack of a reliable assessment of coronary wave speed does not entirely block the study of coronary physiology by applying WIA. Importantly, net WI provides a wealth of information without the need for wave separation. For the sake of argument we will assume that under normal conditions wave speed is $10 \mathrm{~m} / \mathrm{s}$, which is close to the value of wave speed in the aorta and to the values in the coronary circulation as measured in dogs [1]. For the reference vessels and target vessels after stent treatment, coronary pressure will be at a normal level and the total energy in the separated backward and forward waves hardly changes over wide variations of wave speed (Fig. 2). Moreover, as is demonstrated in Fig. 1, the timing of the different waves is hardly affected by the wave speed used, as long as it is reasonably close to physiological values. Marked changes in wave energy were only introduced for simulated wave speeds much below physiological values. Hence, application of the sum-of-squares estimate is not likely to affect conclusions based on relative energy and timing of separated waves in those vessels.

However, the data obtained in stenosed vessels should be interpreted with more caution. As outlined above, coronary wave speed in a vessel segment downstream of the stenosis was likely overestimated to a large degree by the sum-of-squares method. According to Fig. 2 (left panels), this may have resulted in an underestimation of the energy contained in the separated backward and forward waves. Lowering wave speed to more physiological values not only increased the separated wave energies, but also reduced the $\mathrm{B} / \mathrm{F}$ ratio closer to unity in these cases. 
An interesting finding of this study is that the $\mathrm{B} / \mathrm{F}$ ratio in coronary arteries was close to unity in the reference and the treated vessels, suggesting that the backward waves actively generated in the microcirculation were of similar energy as those originating from changes in aortic pressure. Waves in the aorta are predominantly forward, with only small contributions from reflected backward waves. The $\mathrm{B} / \mathrm{F}$ ratio in the aorta is therefore likely much smaller than 1 , but may well increase with coarctation due to larger reflected backward waves [26]. It is plausible that a coronary stenosis affects the $\mathrm{B} / \mathrm{F}$ ratio in a similar fashion as an aortic coarctation, but not through increased backward reflection but via reduced transmission of forward wave energy through the stenosis. However, the uncertainty about coronary wave speed in the presence of a stenosis makes further deductions rather speculative. The fact remains that the $\mathrm{B} / \mathrm{F}$ ratio in coronary vessels by far exceeds that in peripheral vessels, which may point to an interesting property with potential diagnostic applications.

\subsection{Limitations of WIA in appraising myocardial perfusion}

It is clear that WIA may be rather important in advancing our insights in the cause and effect relations between biomechanical events related to heart contraction and aortic pressure on the one hand and the phasic patterns of coronary pressure and flow velocity waveforms on the other hand, and how these are affected by disease. To this end, WIA has been used to investigate the coronary systolic flow impediment [25] and differences between flow waveforms in left and right coronary vessels [6], with emphasis on the influence of backward waves originating from the microcirculation. Nonetheless, the usefulness of WIA to improve our understanding of myocardial perfusion throughout a heartbeat has yet to be demonstrated.

Wave intensity analysis is per definition constrained to information associated with a change in pressure and velocity occurring at distinct moments in time, while myocardial perfusion is largely determined by the mean pressure averaged over the cardiac cycle and coronary resistance. It is conceivable that the same WI profiles are obtained at different beat-average flow rates. Hence, inferences on perfusion of the heart based on the size of the backward expansion wave responsible for flow acceleration at the start of diastole may be erroneous. It is therefore even more unlikely that WIA will be capable to reveal regional or transmural differences in perfusion [8] The continuous changes in intramyocardial pressure and myocardial elastance also affect WI [20]. Moreover, it is well known that myocardial perfusion during hyperemic conditions depends on the diastolic time fraction and decreases inversely with heart rate [5]. Functional studies to address these fundamental questions of coronary-cardiac interaction by applying WIA in humans have yet to be performed.

\section{Conclusions}

Wave intensity is a powerful tool to investigate the pulsatile nature of coronary hemodynamic waveforms in more detail than was possible so far. The relative insensitivity of wave separation on wave speed, at least for values within a physiological range, is an important observation since it enables the separation of traveling waves in the coronary arteries into forward and backward components and therefore allows for a better understanding of aortic, left ventricular and microcirculatory interactions in the coronary circulation. The ratio of integrated backward and forward waves may turn out to be a useful parameter in this scenario.

Acknowledgment This study was supported in part by the Netherlands Heart Foundation (grants 2000.090 and 2006B186).

Open Access This article is distributed under the terms of the Creative Commons Attribution Noncommercial License which permits any noncommercial use, distribution, and reproduction in any medium, provided the original author(s) and source are credited.

\section{References}

1. Arts T, Kruger TI, Van Gerven W, Lambregts JAC, Reneman RS (1979) Propagation velocity and reflection of pressure waves in the canine coronary artery. Am J Physiol Heart Circ Physiol 237:H469-H474

2. Bleasdale RA, Parker KH, Jones CJH (2003) Chasing the wave. Unfashionable but important new concepts in arterial wave travel. Am J Physiol Heart Circ Physiol 284:H1879-H1885

3. Davies JE, Whinnett ZI, Francis DP, Willson K, Foale RA, Malik IS, Hughes AD, Parker KH, Mayet J (2006) Use of simultaneous pressure and velocity measurements to estimate arterial wave speed at a single site in humans. Am J Physiol Heart Circ Physiol 290:H878-H885. doi:10.1152/ajpheart.00751.2005

4. Davies JE, Whinnett ZI, Francis DP, Manisty CH, Aguado-Sierra J, Willson K, Foale RA, Malik IS, Hughes AD, Parker KH, Mayet J (2006) Evidence of a dominant backward-propagating "suction" wave responsible for diastolic coronary filling in humans, attenuated in left ventricular hypertrophy. Circulation 113:17681778. doi:10.1161/CIRCULATIONAHA.105.603050

5. Fokkema DS, VanTeeffelen JWGE, Dekker S, Vergroesen I, Reitsma JB, Spaan JAE (2005) Diastolic time fraction as a determinant of subendocardial perfusion. Am J Physiol Heart Circ Physiol 288:H2450-H2456. doi:10.1152/ajpheart.00790. 2004

6. Hadjiloizou N, Davies JE, Malik IS, Aguado-Sierra J, Willson K, Foale RA, Parker KH, Hughes AD, Francis DP, Mayet J (2008) Differences in cardiac microcirculatory wave patterns between the proximal left mainstem and proximal right coronary artery. Am J Physiol Heart Circ Physiol 295:H1198-H1205. doi: 10.1152/ajpheart.00510.2008

7. Hoffman JI, Spaan JA (1990) Pressure-flow relations in coronary circulation. Physiol Rev 70:331-390 
8. Hoffman JI, Chilian WM (2000) Brief commentary on coronary wave-intensity analysis. J Appl Physiol 89:1633-1635

9. Khir AW, O'Brien A, Gibbs JS, Parker KH (2001) Determination of wave speed and wave separation in the arteries. J Biomech 34:1145-1155. doi:10.1016/S0021-9290(01)00076-8

10. Khir AW, Parker KH (2002) Measurements of wave speed and reflected waves in elastic tubes and bifurcations. J Biomech 35:775-783. doi:10.1016/S0021-9290(02)00025-8

11. Khir AW, Swalen MJP, Feng J, Parker KH (2007) Simultaneous determination of wave speed and arrival time of reflected waves using the pressure-velocity loop. Med Biol Eng Comput 45:1201-1210. doi:10.1007/s11517-007-0241-7

12. Kolyva C, Spaan JAE, Piek JJ, Siebes M (2008) Windkesselness of coronary arteries hampers assessment of human coronary wave speed by single-point technique. Am J Physiol Heart Circ Physiol 295:H482-H490. doi:10.1152/ajpheart.00223.2008

13. Kouwenhoven E, Vergroesen I, Han Y, Spaan JA (1992) Retrograde coronary flow is limited by time-varying elastance. Am J Physiol Heart Circ Physiol 263:H484-H490

14. Krams R, Sipkema P, Westerhof N (1989) Varying elastance concept may explain coronary systolic flow impediment. Am J Physiol Heart Circ Physiol 257:H1471-H1479

15. Parker KH, Jones CJ, Dawson JR, Gibson DG (1988) What stops the flow of blood from the heart? Heart Vessels 4:241-245. doi: 10.1007/BF02058593

16. Parker KH, Jones CJ (1990) Forward and backward running waves in the arteries: analysis using the method of characteristics. J Biomech Eng 112:322-326. doi:10.1115/1.2891191

17. Parker KH (2009) An introduction to wave intensity analysis. Med Biol Eng Comput (this issue). doi:10.1007/s11517-0090439-y

18. Savitzky A, Golay MJE (1964) Smoothing and differentiation of data by simplified least squares procedures. Anal Chem 36(8):1627-1639. doi:10.1021/ac60214a047

19. Siebes M, Verhoeff B-J, Meuwissen M, de Winter RJ, Spaan JAE, Piek JJ (2004) Single-wire pressure and flow velocity measurement to quantify coronary stenosis hemodynamics and effects of percutaneous interventions. Circulation 109:756-762. doi:10.1161/01.CIR.0000112571.06979.B2

20. Spaan J, Kolyva C, van den Wijngaard J, ter Wee R, van Horssen P, Piek J, Siebes M (2008) Coronary structure and perfusion in health and disease. Philos Transact A Math Phys Eng Sci 366:3137-3153. doi:10.1098/rsta.2008.0075

21. Spaan JAE, Breuls NPW, Laird JD (1981) Diastolic-systolic flow differences are caused by intramyocardial pump action in the anesthetized dog. Circ Res 49:584-593

22. Spaan JAE (1995) Mechanical determinants of myocardial perfusion. Basic Res Cardiol 90:89-102. doi:10.1007/BF00789439

23. Sugawara M, Niki K, Ohte N, Okada T, Harada A (2009) Clinical usefulness of wave intensity analysis. Med Biol Eng Comput (this issue). doi:10.1007/s11517-008-0388-x

24. Sun YH, Anderson TJ, Parker KH, Tyberg JV (2000) Waveintensity analysis: a new approach to coronary hemodynamics. J Appl Physiol 89:1636-1644

25. Sun YH, Anderson TJ, Parker KH, Tyberg JV (2004) Effects of left ventricular contractility and coronary vascular resistance on coronary dynamics. Am J Physiol Heart Circ Physiol 286:H1590 H1595. doi:10.1152/ajpheart.01100.2001

26. van den Wijngaard J, Siebes M, Westerhof B (2009) Comparison of arterial waves derived by classical wave separation and wave intensity analysis in a model of aortic coarctation. Med Biol Eng Comput (this issue). doi:10.1007/s11517-008-0387-y

27. Verhoeff B-J, Siebes M, Meuwissen M, Atasever B, Voskuil M, de Winter RJ, Koch KT, Tijssen JGP, Spaan JAE, Piek JJ (2005) Influence of percutaneous coronary intervention on coronary microvascular resistance index. Circulation 111:76-82. doi: 10.1161/01.CIR.0000151610.98409.2F

28. Westerhof N, Boer C, Lamberts RR, Sipkema P (2006) Cross-talk between cardiac muscle and coronary vasculature. Physiol Rev 86:1263-1308. doi:10.1152/physrev.00029.2005 